\title{
Induction Motor Centrifugal Blower Health Diagnostic Based on Color Segmentation of Thermal Image and Vibration Signal Feature
}

\author{
Panut Widodo ${ }^{1,}$, Gunawan Dwi Haryadi ${ }^{1}$, and Achmad Widodo ${ }^{1}$ \\ ${ }^{1}$ Departement of Mechanical Engineering Faculty of Engineering, UNDIP, 50275 Semarang, \\ Indonesia
}

\begin{abstract}
The rotating machinery requires condition monitoring which its measurement without being intrusive operation, especially on the equipment needed to continue running. One such machinery is a centrifugal blower induction motor. Infrared thermography and vibration are important and effective technologies to diagnose of health condition it without destructive or disturb of operations. The diagnostics of induction motor are based on the analysis results data onto vibration and processing thermal image. This paper focused on thermography image processing based on color segmentation which it will produce ROI (region of interest) images. The ROI image is extracted based on HSV color and shape feature. Feature extraction is intended to determine value of mean, standard deviation, kurtosis, skewness and entropy HSV and shape features (area, perimeter, metric, and eccentricity). The highest RMS (root mean square) vibration data is used as reference to classify data into normal and abnormal. Parameters that can be used to classify normal and abnormal conditions based on data analysis are standard deviation Hue, kurtosis HS, skewness HSV, entropy HSV and metric.
\end{abstract}

\section{Introduction}

Centrifugal blower is one of the rotating machines used in power plant system. It serves to blow air from outside which is used as cooling for exhaust frame and Gas Turbine shell. This centrifugal blower is driven an induction motor. These parts can cause a vibration and abnormal temperature in the system, and it can make the machine failure. To prevent such condition, it needs to have a condition based monitoring (CBM). CBM is intended to prevent unplanned breakdowns, maximize the plant availability and reduce the danger associated. Condition monitoring of induction motor can be done online and off line, invasive and non-invasive. Invasive and non-invasive methods of CBM are used to measure motor vibration, temperature, speed, torque variations and Motor current stator analysis (MCSA) for detection of motor abnormalities [1].

One of the methods being developed is condition monitoring based on temperature measurement using infrared thermography (IRT)[2]. IRT is non-destructive, non-invasive

*Corresponding author: pntwidodo@gmail.com 
method, an online or offline and non-contact type condition monitoring technique [3]. Health diagnosis of induction motors can be done by evaluating the image pattern generated because of the transformation of input energy into waste heats. IRT can use as a valuable tool to fault diagnosis in induction motors which relying on infrared data combined with the heat transfer theory. IRT has been widely used in various fields like industry, meteorology, environment, medicine, architecture and other technical fields[2].

Image texture evaluation method is one of the methods of image analysis. The primary problem of texture evaluation is the feature extraction that it can describe the particular feature of the image numerically. It can utilize statistical methods of thermal image analysis. This method can be used for condition monitoring and health diagnosis of induction motor [4]. Image segmentation is a crucial part in image processing before implementing the next step such as feature extraction and classification [5][6]. It has many different kinds of methods are histogram threshold, clustering, edge based, region growing, fuzzy, and neural-network methods [7]. Clustering techniques identified analogous clusters of pixels in the feature space like RGB, HSV, and LUV color space [6].

In this paper proposed condition monitoring and health diagnostics of induction motor of the centrifugal blower based on data analysis of thermal images and vibration. Thermal images data processed by color segmentation method that continued feature extraction process by color HSV and shape feature which will produce feature of normal and abnormal condition. Induction motor health diagnostics is based on statistical analysis result of boundary condition of ROI image features.

\section{Basic Theory}

\subsection{Color segmentations}

Segmentation is done to split an image into specific sections for further analysis, resulting in a higher level illustration of the image pixels like objects or foreground and background. Segmentation based on colour in certain cases, is the simplest and most obvious way of distinguishing between objects and background [6]. One of the image segmentation methods is clustering. Clustering techniques identified homogeneous clusters of pixels in the feature space such as RGB and HSV color space [7]. RGB color space is identical with original image colors, so to get HSV needed conversion RGB to HSV color space[5][6].

The RGB colour space consists of three components colors (Red, Green, blue), where all components have 8-bit depth. Every component is interpreted as unsigned integers in range $(0 ; 255)$. It is based on the portion of the electromagnetic spectrum visible to humans (approximate range of wave lengths in 400-700 nm)[5][6].

The HSV color model defines color in terminology of three components : hue $(\mathrm{H})$, saturation (S), and value (V). It is an approximately uniform perceptual color space. Hue is the dominant wavelength of the colour, used to distinguish colors and determine redness, etc. Saturation is the 'purity' of colour (in the sense of the amount of light white mixed with it). Value is the brightness of the colour (also known as luminance) [5][6].

\subsection{Feature extraction of image}

Feature extraction is the process to regain the most important data from the raw data to find parameters that defines the shape of a character exactly and uniquely [8]. It can be classified into 3 groups are color feature extraction, shape and texture features [9]. Extraction feature of HSV and RGB is example color feature extraction. The calculation of the perimeter and area are meaningful for binary images. The binary image containing one 
or more object, where $\mathbf{p}(\mathrm{x}, \mathrm{y})=1$ if a pixel is part of the object or foreground and $\mathbf{p}(\mathrm{x}, \mathrm{y})=$ 0 for background pixels [6]. From area and perimeter can be calculated form factor or called 'Metric'[5]. Eccentricity is the value of comparison between distance between the ellipse minor and major. Eccentricity and metric value have range 0 to 1 . Calculation of eccentricity (e) and metric (M) can be used Equation 1 . Where ' $a$ ' is mayor axis, ' $b$ ' is the minor axis, ' $\mathrm{A}$ ' is area and ' $\mathrm{C}$ ' is the perimeter.

$$
\mathrm{e}=\sqrt{1-\frac{b^{2}}{a^{2}}}, M=\frac{4 \pi A}{C^{2}}
$$

\section{Methodology}

\subsection{Data acquisition process}

Collecting data in this study is conducted by measuring the induction motor centrifugal blower contained in the power generation unit. This is done when the induction motor operates. Specifications of induction motor of the centrifugal blower are $100 \mathrm{HP}, 1180 \mathrm{~A}$, $460 \mathrm{~V}$ AC 3 phase $/ 60 \mathrm{~Hz}$, and diameter motor $411 \mathrm{~mm}$. Thermography images data taken by the infrared thermography (IRT) camera Flir E-40, and vibration using vibscanner.

The infrared camera manufactured by Flir E-40 can measure the temperature on a range scale of $-20{ }^{\circ} \mathrm{C}$ to $650{ }^{\circ} \mathrm{C}$. The thermal lens produces visual image of $640 \times 480$ pixels and captures images of $320 \times 240$ pixels. The distance between induction motor and thermal imager were range of 2.0 metres, with ambient temperature was $35-41{ }^{0} \mathrm{C}$. The emissivity value was set at 0.95 as recommended material of cast iron [2].

Vibration on this object is carried out with 3 positions of horizontal position, vertical and radial at both ends. The result of vibration measurement is Root-Mean Square (RMS in $\mathrm{mm} / \mathrm{s}$ ) data record, while flir E-40 produces thermal image $320 \times 240$ pixels. This thermal image will be crop to get the observed region of interest (ROI).

\subsection{Data classifications, HSV color and shape feature extraction}

Classification data based on vibration data according to ISO 10816-3 standard. After cropping, the thermal image implemented RGB color segmentation by K-mean clustering to produce of Region of interest (ROI) image. The pixel features are clustered to group them into 4 regions of similar color. From number 4 cluster images selected one image of ROI representing temperature increases that is redness image region. This ROI calculated of HSV characteristic (mean, standart deviation, kurtosis, skewness and entropy) and shape feature (area, perimeter and eccentricity). Image statistics are investigated and studied based on first order statistics that capture certain statistical regularities of ROI thermal image [10], following Equation 2-5, where $\sum \sum \mathrm{p}(\mathrm{x}, \mathrm{y})$ represents the summation of all pixel values of the component $\mathrm{HSV}$ and $\mathrm{MxN}$ is size of the image.

$$
\begin{gathered}
\text { Mean }(\mu)=\frac{1}{M x N} \sum_{x=1}^{M} \sum_{y=1}^{N} p(x, y), \text { Std.dev. }(\sigma)=\sqrt{\frac{1}{M x N} \sum_{x=1}^{M} \sum_{y=1}^{N}(p(x, y)-\mu)^{2}} \\
\text { Entropy }\left(E_{n}\right)=\frac{1}{M x N} \sum_{x=1}^{M} \sum_{y=1}^{N} p(x, y) \log _{2}(p(x, y)) \\
\text { Skewness }(s)=-\frac{1}{M x N} \sum_{x=1}^{M} \sum_{y=1}^{N}\left|\frac{p(x, y)-\mu}{\sigma}\right|^{3}
\end{gathered}
$$




$$
\operatorname{Kurtosis}(k)=-\frac{1}{M x N} \sum_{x=1}^{M} \sum_{y=1}^{N}\left|\frac{p(x, y)-\mu}{\sigma}\right|^{4}-3
$$

Next step is plotting the features of statistical HSV and shape feature. Afterwards selected features that can distinguish between normal and abnormal conditions. Final step is determining the boundary condition between normal and abnormal using statistical logic method. Boundary values obtained by calculation value of mean, min, max, standard deviation, delta (D), final minimum and final maximum of selected feature data.Value of delta (D) is average difference between minimum and maximum value in each class.

$$
\text { final } \max =\max +D, \text { final } \min =\min -D
$$

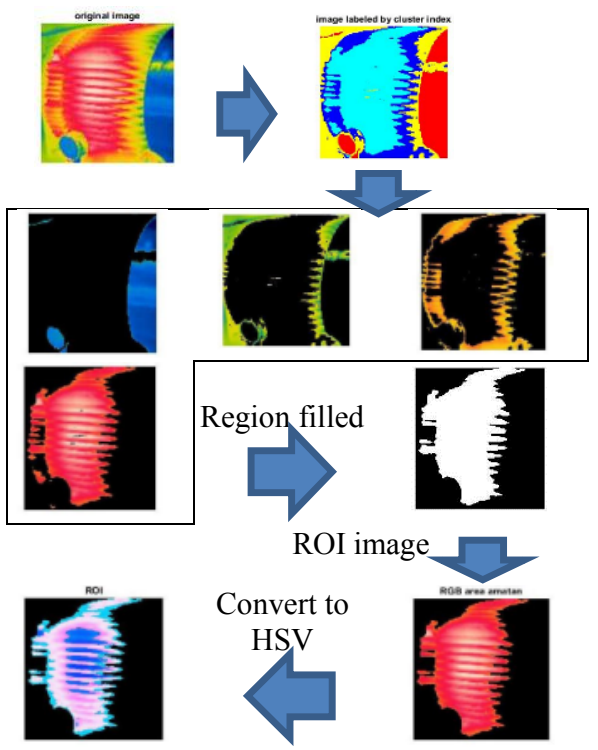

(a)

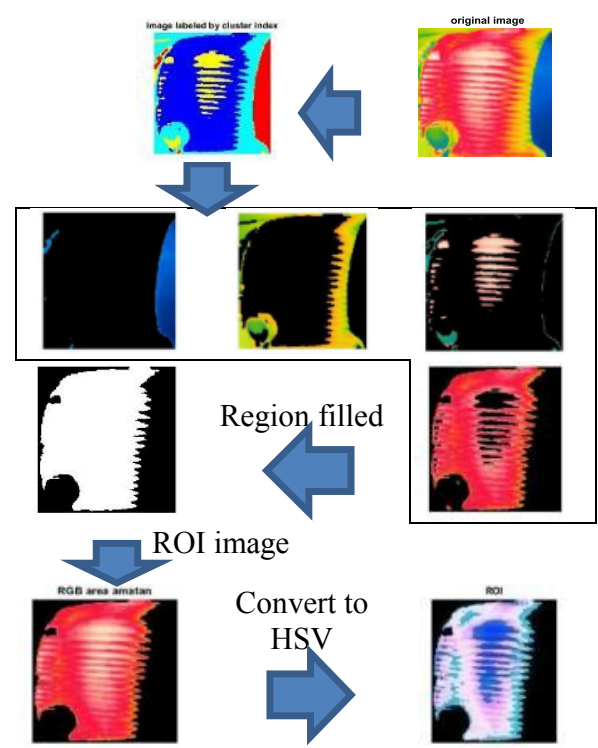

(b)

Fig. 1. Color segmentation process, decide to ROI: (a) Normal condition, (b) Abnormal condition

\section{Result and Discussion}

Induction motor of the centrifugal blower used as subject of this observation according to ISO 10816-3 can be categorized into a group 1 Rigid. Classification based on motor diameter $411 \mathrm{~mm}$ (motor diameter more than $315 \mathrm{~mm}$ ). Normal condition if the root mean square (RMS) values has up $2.3 \mathrm{~mm} / \mathrm{s}$. If then RMS $>2.3 \mathrm{~mm} / \mathrm{s}$ being categorized as abnormal condition. From number 50 data can be classified into number 40 data of normal and 10 data of abnormal condition. Feature of the highest vibration shown in Figure 2a.

The ROI image determination process and convert to HSV image shown in Figure 1. ROI, which is an RGB image converted into HSV image, extracted to calculate the statistical of each component of HSV. ROI will be transformed into binary image by threshold method, then extracted to calculate area, perimeter and eccentricity. Referring to determination and the resulting feature, not all features can be used as reference to differentiate induction motor conditions. Figure $2 b$ shows random patterns for mean HSV of hot area of ROI which are not precise patterns because the conditions of motor induction cannot be distinguished clearly. Feature mean of HSV has similarity between normal and abnormal condition. As well as standard deviation of $\mathrm{S}$ and $\mathrm{V}$, kurtosis $\mathrm{V}$, area, perimeter 
and eccentricity features. Feature that can differentiate its condition attend standard deviation of Hue, kurtosis HS, skewness HSV, entropy HSV and metric.

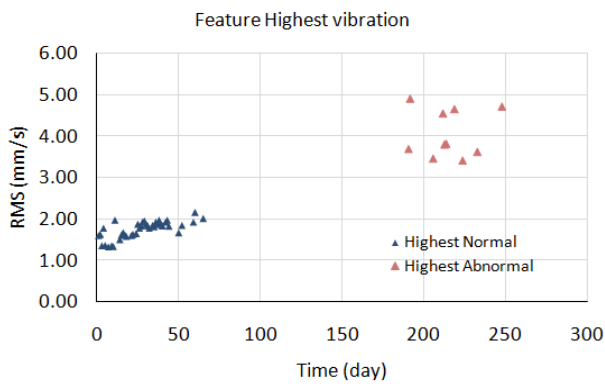

(a)

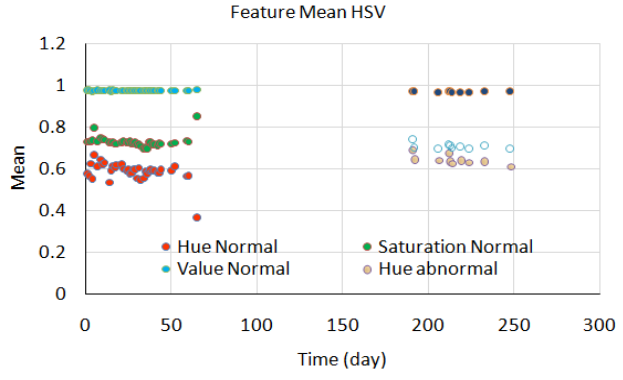

(b)

Fig. 2.Feature; (a) Result of highest vibration, (b) Mean of each component HSV

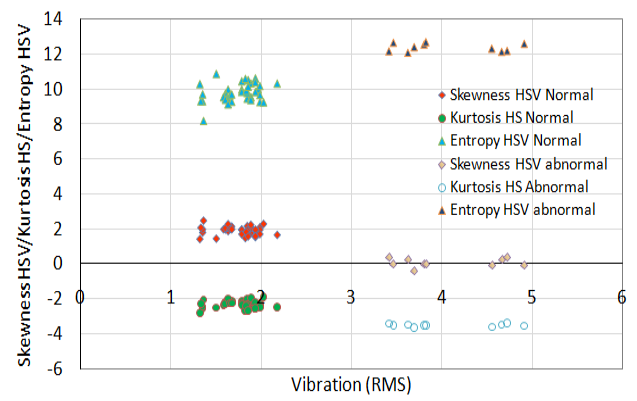

a. Feature vibration - kurtosis HS/skewness HSV/entropy HSV (KSE HSV)

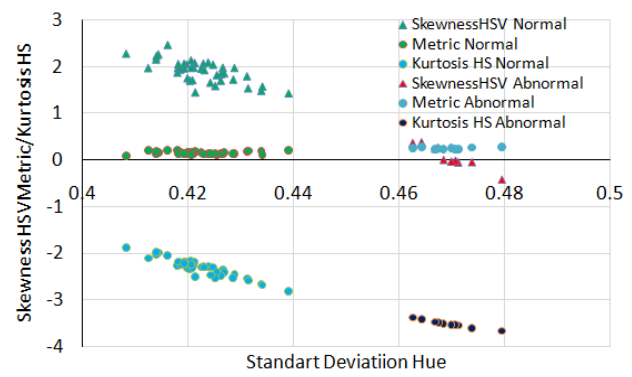

c.Feature SD Hue - skew HSV/metric/kurt HS

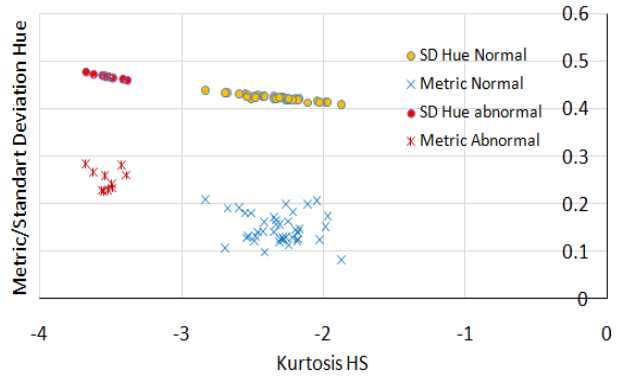

b. Feature kurtosis HS - metric or SD Hue

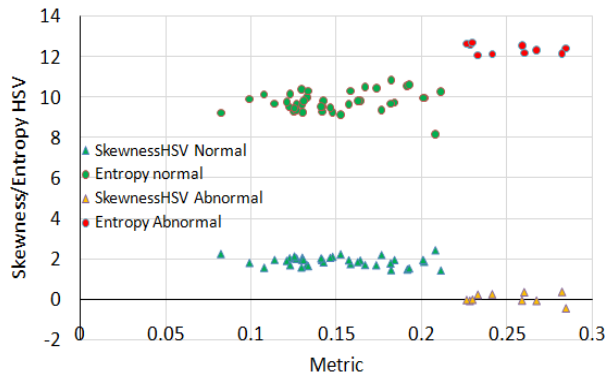

d.Feature metric - skewness or entropy HSV

Fig. 3.Feature differentiate between normal and abnormal condition

HSV parameter values obtained by summing the component values of $\mathrm{H}, \mathrm{S}$ and $\mathrm{V}$, like skewness HSV is skewness value of component $\mathrm{H}, \mathrm{S}$ and $\mathrm{V}$. While for kurtosis HS obtained from sum of the kurtosis $\mathrm{H}$ and kurtosis $\mathrm{S}$ value. In order to determine the good features to be used as reference differentiate of normal and abnormal conditions, it is necessary to simulate the plot of each feature parameter. Not only juxtaposed with time only. A good feature pattern is shown in Figure 3.a-d feature pattern RMS-KSE HSV, RMS-SD H, RMS-Metric, kurtosis HS-Metic etc. Along with the addition of vibration value then the value of entropy, standard deviation hue, metric will rise while for kurtosis and skewness will decrease towards negative value.

Finally, the result of determination of boundary conditions value (final maximum/F.max and minimum/F.min) according Equation 6, can be shows Table 1. Not overlapping value of boundary condition between normal and abnormal condition. 
Table 1.Determination result of boundary condition value of each parameters.

\begin{tabular}{|c|c|c|c|c|c|c|c|c|c|c|c|c|}
\hline \multirow{2}{*}{$\begin{array}{l}\text { Condition of } \\
\text { induction motor }\end{array}$} & \multicolumn{2}{|c|}{ RMS } & \multicolumn{2}{|c|}{ SD Hue } & \multicolumn{2}{|c|}{ Skewness HSV } & \multicolumn{2}{|c|}{ Kurtosis HS } & \multicolumn{2}{|c|}{ Entropy HSV } & \multicolumn{2}{|c|}{ Metric } \\
\hline & F.Min & F.Max & F.Min & F.Max & F.Min & F.Max & F.Min & F.Max & F.Min & F.Max & F.Min & F.Max \\
\hline Normal & 1.1125 & 3885 & 0.4009 & 0.4443 & 1447 & 2.6523 & -3.0072 & -1.63 & 7.9164 & 11.6549 & 0.0539 & 0.2122 \\
\hline Abnormal & 2.7370 & 5.3910 & 0.4562 & 0.4829 & -0.5850 & 0.7187 & -3.7544 & -3.2926 & 11.8459 & 12.9362 & 0.2262 & 0.3027 \\
\hline
\end{tabular}

\section{Conclusion}

Based on this study, HSV color segmentation on the thermal image can be used to diagnose normal and abnormal conditions of induction motor. Parameters that can distinguish between normal and abnormal conditions are standard deviation Hue, skewness HSV, kurtosis HS, entropy HSV and metric. Induction motor of centrifugal blower is categorized as abnormal condition if RMS $>2.3885 \mathrm{~mm} / \mathrm{s}$, standard deviation Hue $\geq 0.4562$, skewness $\mathrm{HSV} \leq 0.7187$, Kurtosis HS $<-3.0072$, Entropy HSV $\geq 11.8459$, and metric $\geq 0.2262$.

The author gratefully acknowledges the support of measuring instruments and research objects from PT. Indonesia Power Semarang on this research

\section{References}

[1] D. G. Ece and M. Başaran, "Condition monitoring of speed controlled induction motors using wavelet packets and discriminant analysis,"Expert System Application", vol. 38, pp. 8079-8086 (2011)

[2] S. Bagavathiappan, B. Lahiri, T. Saravanan, J. Philip, and T. Jayakumar, "Infrared thermography for condition monitoring, A review," Infrared Phys. Technol., vol. 60, pp. 35-55 (2013)

[3] M. S. Jadin and S. Taib, "Recent progress in diagnosing the reliability of electrical equipment by using infrared thermography," Infrared Phys. Technol., vol. 55, pp. 236-245 (2012)

[4] M. Fidali, "An idea of continuous thermographic monitoring of machinery, " in Proceedings of the 2008 International Conference on Quantitative Infrared Thermography, pp. 61-18 (2008)

[5] C. Solomon and T. Breckon, Fundamentals of Digital Image Processing: A Practical Approach with Examples in Matlab, vol. 3. Chichester, UK: John Wiley \& Sons, Ltd, (2010)

[6] R. Gonzalez and R. Woods, Digital image processing, (2002)

[7] H. Wang and D. Suter, "Color Image Segmentation Using Global Information and Local Homogeneity," Proceeding VIIth Digital Image Computing: Techniques and Applications, pp. 10-12 (2003)

[8] G. Kumar and P. K. Bhatia, "A detailed review of feature extraction in image processing systems," in Fourth International Conference on Advanced Computing and Communication Technologies. IEEE Computer Society, pp. 5-12 (2014)

[9] S. A. Medjahed, "A Comparative Study of Feature Extraction Methods in Images Classification,” I.J. Image, Graph. Signal Process. 7, 3 (2015)

[10] S. Selvarajah and S. R. Kodituwakku, "Analysis and Comparison of Texture Features for Content Based Image Retrieval," International Jurnal Latest Trends Computing 2, 1 (2011) 